\title{
КООРДИНАЦИОННЫЕ ЧИСЛА ПЕРИОДИЧЕСКИХ И КВАЗИПЕРИОДИЧЕСКИХ СТРУКТУР
}

https://doi.org/10.31241/MIEN.2018.14.15

\author{
Шутов А.В. ${ }^{1}$, Малеев А.В. ${ }^{2}$, Кежутина O.B. ${ }^{3}$
}

${ }^{1}$ Владимирский государственный университет, Владимир, a1981@mail.ru

${ }^{2}$ Владимирский государственный университет, Владимир, andr_mal@mail.ru

${ }^{3}$ Владимирский государственный университет, Владимир, sakura.1004@yandex.ru

\section{Аннотация}

Координационные числа представляют собой одну из важнейших характеристик кристаллических и квазикристаллических структур. В работе приводится обзор результатов о координационных числах периодических и квазипериодических разбиений и графов. В периодическом случае сформулирована общая гипотеза, описывающая поведение координационных чисел, а также приведены три общих подхода, позволяющих получать явные формулы. В квазипериодическом случае обсуждаются общие методы получения асимптотических формул для координационых чисел. Для двух конкретных квазипериодических структур - разбиения Рози и графа вершин разбиения Пенроуза - соответствующие асимптотические формулы приводятся в явном виде, и проводится исследование отклонений реальных координационных чисел от главных членов их асимптотик.

\section{Summary}

Coordination numbers are one of the most important characteristics of crystalline and quasicrystalline structures. In this paper we review the results on the coordination numbers of periodic and quasiperiodic tilings and graphs. In the periodic case, we state a general conjecture describing a behavior of coordinate numbers, and discuss three general approaches to obtain explicit formulas. In the quasiperiodic case, general methods for obtaining asymptotic formulas for coordination numbers are discussed. For two concrete quasiperiodic structures: Rauzy tiling and vertex graph of Penrose tiling, the corresponding asymptotic formulas are given in explicit form and the deviations of the real coordination numbers from the principal terms of their asymptotics are studied.

Одной из важнейших топологических характеристик любой кристаллической структуры является последовательность ее координационных чисел (координационная последовательность), определяемых как число атомов или молекул структуры, принадлежащих последовательности координационных сфер некоторого фиксированного атома или молекулы. Координационные сферы обычно определяются индуктивно. Первая координационная сфера состоит из всех атомов/молекул, соседних с выбранным. $n$-ая сфера состоит из атомов/молекул, соседних с атомами/молекулами (n-1)-ой координационной сферы, не входящих в координационные сферы с номерами, меньшими $n$. Концепция координационной последовательности была введена в работе [1]. В дальнейшем координационные числа активно изучались для различных кристаллических структур, в первую очередь для цеолитов [2-5]. 
Для математического описания координационной последовательности обычно используется модель, представляющая структуру в виде периодического графа, вершины которого соответствуют атомам или молекулам структуры, а ребра - связям между ними. При этом оказывается естественным рассматривать периодические графы в пространстве произвольной размерности $d$.

На произвольном графе имеется естественная функция расстояния $d(x, y)$, определяемая как число ребер в кратчайшей цепи графа, соединяющей вершины $x$ и $y$. При помощи этой функции $n$-ую координационную сферу вершины $x$ можно определить, как множество вершин графа, расположенных на расстоянии $n$ от $x$.

$$
e q(x, n)=\{y: d(x, y)=n\}
$$

Координационные числа $e(x, n)$ при этом определяются как числа вершин графа в $e q(x, n)$.

Вместо графов можно рассматривать и другие модели кристаллических структур, такие как разбиения или упаковки с заданным на них отношением соседства [6].

Изучение координационных последовательностей различных структур [7-14] привели к следующей гипотезе, впервые сформулированной в [15]. Пусть $G$ - периодический граф размерности $d, e(x, n)$ - его $n$-е координационное число для вершины $x$. Тогда существуют многочлены $P_{l}(n), \ldots, P_{k}(n)$ степени $d-1$ с рациональными коэффициентами, такие, что при $n \geq n_{0}$ выполняется равенство $e(x, n)=P_{i}(n)$, где $n=i(\bmod k)$. Данная гипотеза полностью описывает поведение координационных чисел в периодическом случае.

В качестве альтернативного описания можно использовать производящую функцию $G F(z)=\sum_{n \geq 0} e(x, n) z^{n}$. В этом случае утверждается, что производящая функция рациональна и представима в виде $G F(z)=\frac{\sum_{i=0}^{m-1} c_{i} z^{i}}{\prod_{i=0}^{k}\left(1-z^{d_{i}}\right)} \cdot$

Отметим, что сами координационные числа при этом могут быть найдены при помощи разложения производящей функции $G F(z)$ в ряд Тейлора. Отметим, что предложенные описания координационных последовательностей эквивалентны между собой.

В качестве примера на рис. 1 изображено поведение координационных чисел для сетки Лавеса L3C.

Сами координационные числа в этом случае описываются формулой

$$
e(x, n)=\left\{\begin{array}{c}
(5 n-4) / 2, n \equiv 0(\bmod 4) \\
(9 n+3) / 4, n \equiv 1(\bmod 4) \\
2 n+2, n \equiv 2(\bmod 4) \\
(9 n-3) / 4, n \equiv 3(\bmod 4)
\end{array} .\right.
$$



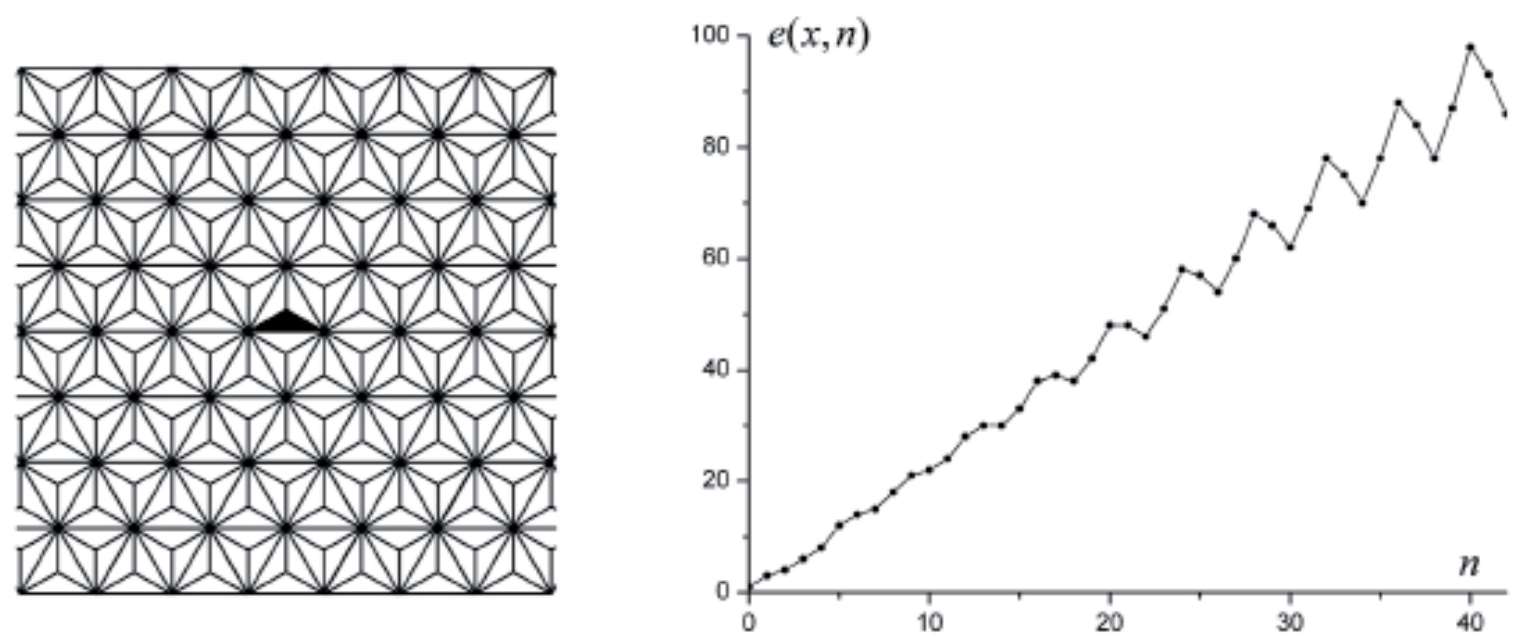

Pис. 1. Сетка Лавеса L3C и ее координационные числа.

В настоящее время существует ряд общих подходов, видимо позволяющих получить формулы, описывающие координационную последовательность произвольной периодической структуры. Тем не менее, следует отметить, что полное доказательство сформулированной выше гипотезы в настоящее время нигде не опубликовано.

Первый из таких подходов [16-17] основывается на рассмотрении факторграфа, состоящего из вершин и ребер исходного графа, неэквивалентных относительно решетки трансляций, с сохранением отношения инцидентности. На множестве, порожденном формальными линейными комбинациями цепей этого факторграфа, вводится операция умножения, превращающая его в коммутативное и ассоциативное кольцо с единицей. При этом задача вычисления производящей функции координационной последовательности сводится к задаче вычисления обратного элемента для некоторого специально построенного элемента кольца.

Альтернативный подход [18-19] основан на изучении геодезических в периодическом графе. Игнорируя некоторые технические детали, для произвольной вершины $x$ в $d$-мерном периодическом графе $G$ имеется специальная геодезическая $\Gamma$, представимая в виде $\Gamma: \Gamma_{1} \rightarrow k_{1} p_{1} \rightarrow \ldots \rightarrow k_{d} p_{d}$, где $k_{l}$ - целые неотрицательные числа, цепи $p_{1}$ начинаются и заканчиваются в вершинах, сравнимых по модулю решетки периодов и больше не содержат пар вершин, сравнимых по модулю этой решетки (такие цепи называются лучами), а длина цепи $\Gamma_{1}-$ не превосходит числа вершин в фундаментальной области графа. [20]. Обратно, все цепи такого вида оказываются геодезическими. Число цепей рассматриваемого вида длины $n$ равно $r\left(\left|p_{1}\right|, \ldots,\left|p_{d}\right|, n-\left|\Gamma_{1}\right|\right)$, где $r\left(a_{1}, \ldots, a_{d}, n\right)$ - число решений уравнения

$$
a_{1} x_{1}+\ldots+a_{d} x_{d}=n
$$

в целых неотрицательных числах $x_{1}, \ldots, x_{m}$. Для функции $r\left(a_{1}, \ldots, a_{d}, n\right)$ известно [21], что при $A=H O K\left(a_{1}, \ldots, a_{d}\right)$ функция $r\left(a_{1}, \ldots, a_{d}, A n+b\right)(0 \leq b<A)$ является многочленом степени $d-1$ относительно $n$. Кроме того, для ее производящей функции справедливо соотношение 


$$
\sum_{n=1}^{\infty} r\left(a_{1}, \ldots, a_{d}, n\right) t^{n}=\frac{1}{\left(1-t^{a_{1}}\right) \ldots\left(1-t^{a_{d}}\right)}
$$

Таким образом, вычисление координационных чисел сводится к перебору лучей $p_{i}$ и цепей $\Gamma_{1}$ с последующим решением линейных диофантовых уравнений.

Еще один подход к изучению координационных чисел в периодическом случае, основан на более глубоком изучении координационных сфер. Согласно [19-20] для любого $d$-мерного периодического графа $G$ существует многогранник $p l_{G}$ (многогранник роста графа) такой, что координационная сфера $e q(x, n)$ лежит в $C$-окрестности многогранника $x+n \cdot p o l_{G}$, причем постоянная $C$ не зависит от номера координационной сферы. Многогранник $\mathrm{pol}_{G}$ естественным образом разбивает $d$-мерное пространство на ряд секторов и позволяет определить секторные координационные сферы $e q_{i}(x, n)$, как фрагменты координационных сфер $e q(x, n)$, попадающие в соответствующие сектора, а также секторные координационные числа $e_{i}(x, n)$, равные числам вершин графа в соответствующих секторных координационных сферах. При этом оказывается, что сферы $e q_{i}(x, n)$ обладают свойством периодичности. Другими словами, их можно рассматривать как множества точек из конечного числа $(d-1)$-мерных решеток, попадающих в заданные области $E_{i}(n)$. При этом типы соответствующих решеток зависят только от остатка от деления $n$ на некоторое число $A$, зависящее от графа $G$ и от номера сектора. При этом если $n_{1} \equiv n_{2}(\bmod A)$, то соответствующие фигуры $E_{i}\left(n_{1}\right)$ и $E_{i}\left(n_{2}\right)$ оказываются гомотетичными. Таким образом, задача о вычислении координационных чисел сводится к конечному числу задач о вычислении функций $r(n ; L, E)$ где $r(n ; L, E)$ - число точек решетки $L$, попавших в область $n E$. Данные функции являются полиномиальными (многочлены Эрхарта). Подробности об их вычислении можно найти в [22].

В последние десятилетия одной из актуальных задач современной кристаллографии является построение и изучение математических моделей квазикристаллических структур. В связи с этим представляет интерес вопрос о поведении координационных последовательностей реальных и математических квазикристаллов.

В этом случае, по все видимости, не существует явных формул для координационных чисел $e(x, n)$, поэтому актуальной является задача получения приближенных асимптотических формул. Отметим, что в периодическом случае требуемая асимптотическая формула имеет вид

$$
e(x, n)=c(n \bmod k) n^{d-1}+O\left(n^{d-2}\right)
$$

где рациональные числа $c(n \bmod k)$ описывают старшие коэффициенты многочленов $P_{i}(n)$.

Одним из самых эффективных подходов к построению и изучению математических квазипериодических структур является подход, при котором 
всем объектам квазипериодической структуры ставятся в соответствие некоторые двойственные объекты в пространстве параметров. При этом сложность квазипериодической структуры измеряется размерностью пространства параметров. Примерами структур с одномерным пространством параметров являются разбиение Рози [23] и разбиения Ито-Оцуки [24]. Двумерное пространство параметров возникает при изучении разбиения Пенроуза [25-26] и графа его вершин [27-29].

Из всех подходов, применявшихся к изучению координационных чисел периодических структур, на случай квазикристаллов удается частично перевести только аналог подхода, основанного на анализе секторных координационных окружений. В случае квазипериодических разбиений и графов удается получать аналоги теоремы о форме роста, что позволяет определить аналоги секторных координационных окружений и секторных координационных чисел. При этом оказывается, что вершины из секторных координационных окружений $e q_{i}(x, n)$ при фиксированном $n$ лежат в конечном наборе подпространств размерности $d$-1, параллельных определяющей сектор грани многогранника роста. Тем самым, секторные координационные окружения фактически можно рассматривать как квазикристаллические структуры размерности $d-1$. Эти структуры допускают параметризацию, получаемую некоторым сечением параметризации исходной квазипериодической структуры. Таким образом, для вычисления координационных чисел требуется уметь решать задачу о числе точек квазипериодической структуры, попадающих в гомотетично растягиваемые области. Эта задача фактически эквивалентна задачи вычисления плотности множества вершин квазипериодической структуры.

В случае $d=2$ секторные координационные сферы $e q_{i}(x, n)$ и соответствующие им множества параметров оказываются одномерными. При этом движение вдоль секторной координацинной сферы приводит к построению некоторого отображения $T$, действующего на множестве параметров секторной координационной сферы. Данное отображение обычно оказывается перекладыванием отрезков. Изучение свойств этого перекладывания позволяет связать длину секторной координационной сферы (вычисляемую при помощи теоремы о форме роста) с числом вершин в ней, что и дает требуемую асимптотическую формулу для координационных чисел.

При этом если пространство параметров исходной квазипериодической структуры было одномерным, то параметризация секторных координационных окружений $e q_{i}(x, n)$ и вид отображения $T$ оказываются не зависящими от $x$ и $n$ и имеет место асимптотическая формула

$$
e(x, n)=c n+o(n)
$$

При этом, в отличие от периодического случая, коэффициент с оказывается иррациональным. Например, в случае разбиения Рози получается [23] $=5+2 \zeta+5 \zeta^{2}$, где $\zeta-$ единственный вещественный корень кубического уравнения $x^{3}+x^{2}-x=1$. 
Если же исходное пространство параметров имело размерность, большую единицы, различным $x$ и $n$ соответствуют различные параметризации секторных координационных окружений $e q_{i}(x, n)$. Это приводит к тому, что асимптотическая формула для координационных чисел принимает вид

$$
e(x, n)=c(x, n) n+o(n)
$$

где $c(x, n)$ - некоторая достаточно сложная функция, принимающая бесконечно много значений.

Например, в случае графа вершин разбиения Пенроуза получаем

где $\quad F(n, \varepsilon, \delta)=\left\{\begin{array}{l}\tau^{-1}-\left(\frac{1}{2}-\tau^{-3}\right)\left\{\varepsilon \frac{n-1}{2} \tau^{-2}+\delta\right\},\left\{\varepsilon \frac{n-1}{2} \tau^{-2}+\delta\right\} \in\left[0 ; \tau^{-1}\right) \\ \frac{\tau^{-1}}{2}+\tau^{-3}-\frac{1-\tau^{-3}}{2}\left\{\varepsilon \frac{n-1}{2} \tau^{-2}+\delta\right\},\left\{\varepsilon \frac{n-1}{2} \tau^{-2}+\delta\right\} \in\left[\tau^{-1} ; 1\right)\end{array}\right.$.

Здесь числа $\varepsilon_{i} \pm 1$ и $\delta_{i}$ зависят от выбора начальной вершины $x$, а также от четности/нечетности $n, \tau=\frac{1+\sqrt{5}}{2}$, а $\{x\}-$ дробная доля $x$. При этом зависимость от четности/нечетности $n$ обусловлена различиями в параметризации соответствующих секторных координационных окружений $e q_{i}(x, n)$.

В частности, если начальная вершина $x$ совпадает с началом координат, получаются следующие результаты.

1) если $n$-нечетно и $\left\{\frac{n-1}{2} \tau^{-2}\right\} \in\left[0 ; \tau^{-1}\right)$, то $c(O, n)=10 \tau^{-2}+\frac{5}{2} \tau^{-1}$,

2) если $n$-нечетно и $\left\{\frac{n-1}{2} \tau^{-2}\right\} \in\left[\tau^{-1} ; 1\right)$, то $c(O, n)=10 \tau^{-2}$,

3) если $n$ - четно и $\left\{\frac{n-2}{2} \tau^{-2}\right\} \in\left[0 ; \tau^{-3}\right)$, то $c(O, n)=5 \tau^{-2}+\frac{25}{2} \tau^{-3}$,

4) если $n$-четно и $\left\{\frac{n-2}{2} \tau^{-2}\right\} \in\left[\tau^{-3} ; \tau^{-1}\right)$, то $c(O, n)=5-\frac{5}{2} \tau^{-3}\left\{\frac{n-2}{2} \tau^{-2}\right\}$,

5) если $n$ - четно и $\left\{\frac{n-2}{2} \tau^{-2}\right\} \in\left[\tau^{-1} ; 1\right)$, то $c(O, n)=5-5 \tau^{-4}+\frac{5}{2} \tau^{-3}\left\{\frac{n-2}{2} \tau^{-2}\right\}$

Соответствующий график координационных чисел $e(O, n)$ изображен на рис. 2.

Отметим, что вычисление координационных чисел квазипериодических структур размерности $d>2$ в настоящее время остается открытой проблемой.

В случае квазипериодических структур представляют также интерес отклонения $r(n)$, описывающие разницу между координационными числами и главными членами соответствующих асимптотических формул $[24,30]$. 


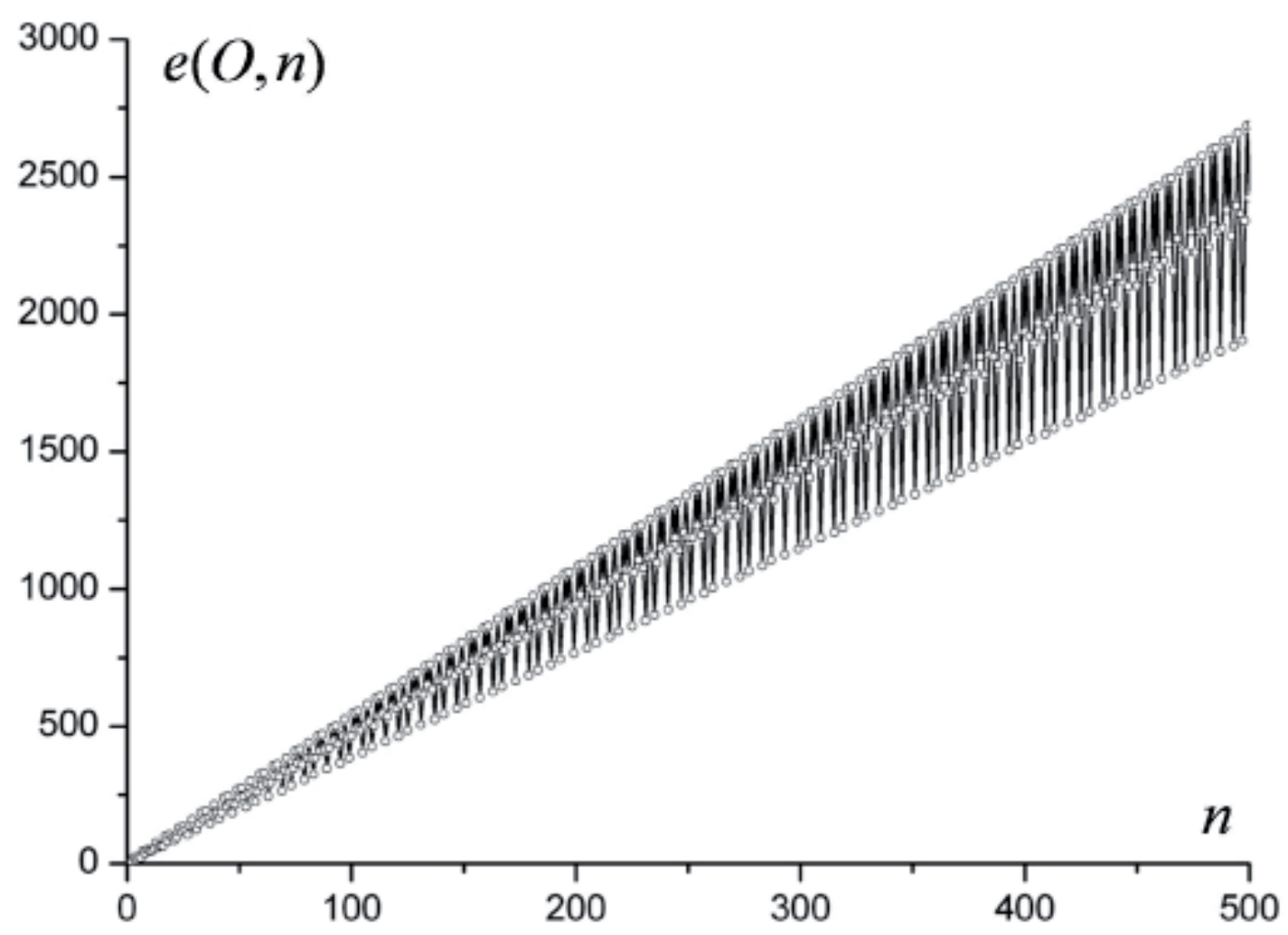

Рис. 2. Координационные числа графа вериин разбиения Пенроуза.

В случае разбиения Рози и разбиений Ито-Оцуки введенные отклонения ведут себя достаточно схожим образом. Проиллюстрируем их поведение на примере разбиения Рози. График функции $r(n)$ в этом случае изображен на рис. 3.

Для изучения сложной функции $r(n)$ она представляется в виде суммы более простых функций $r_{i}(n)$, описывающих отклонения соответствующих секторных координационных чисел. Соответствующие графики для трех из

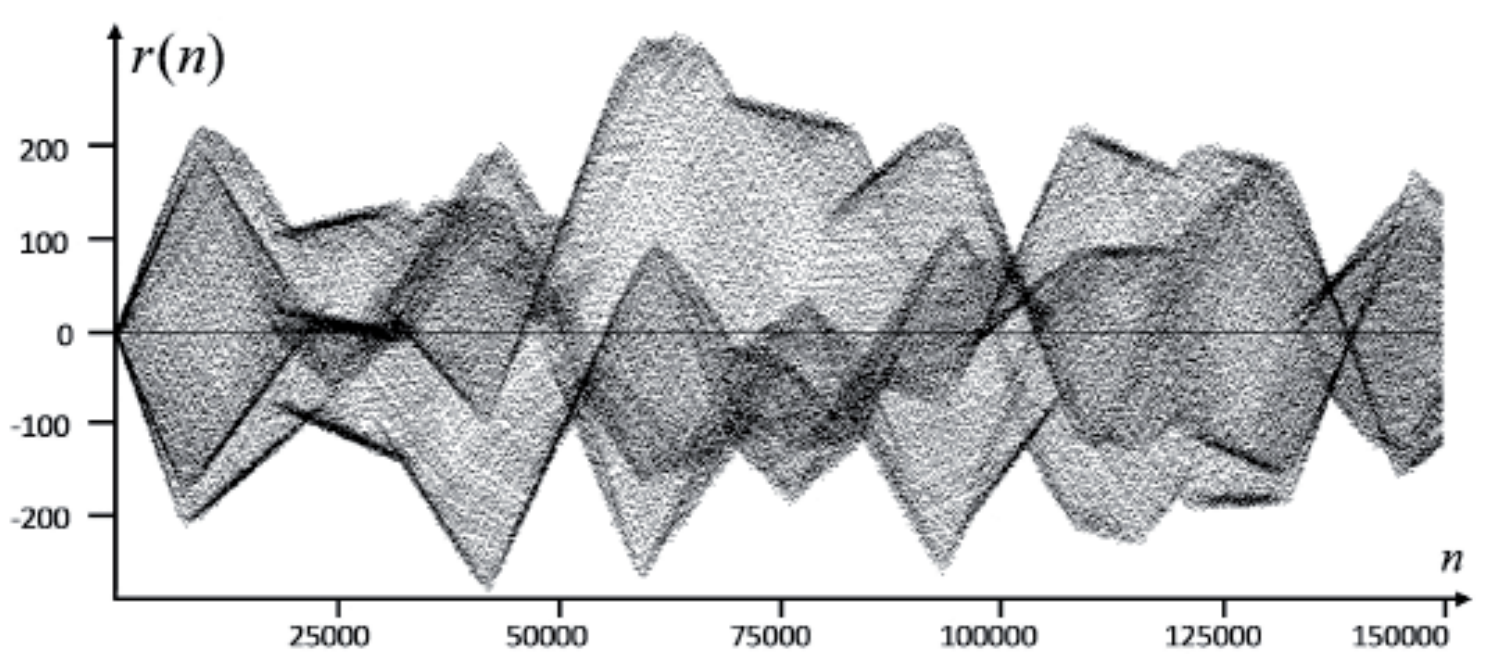

Рис. 3. Отклонения $r(n)$ координационных чисел от главного члена асимптотической формулы для разбиения Рози. 
восьми секторов изображены на рис. 4 (еще 3 функции $r_{i}(n)$ совпадают с изображенными в силу центральной симметрии разбиения Рози, а две оставшиеся - ограниченны).
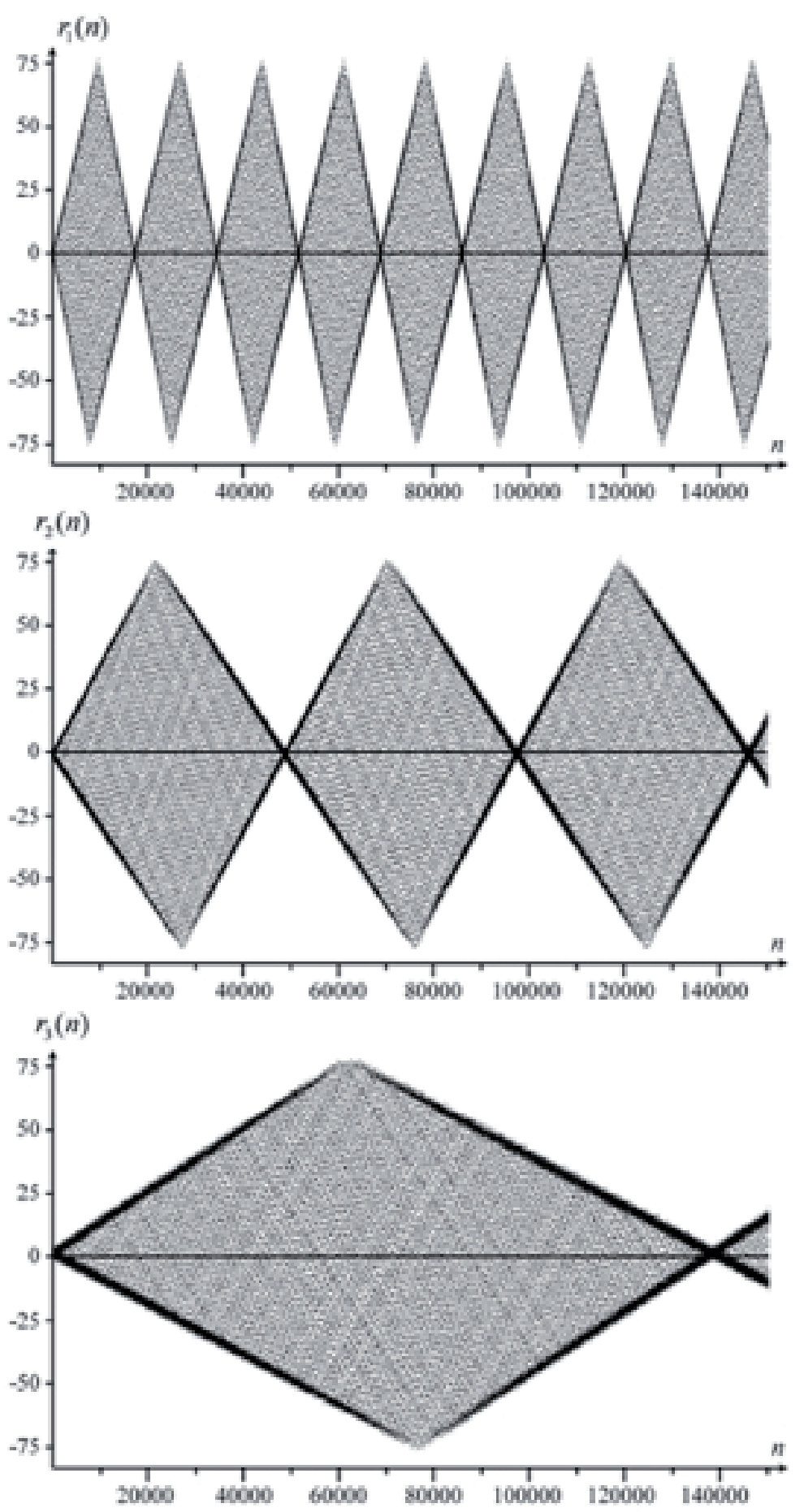

Рис. 4. Отклонения ri(n) секторных координационных чисел трех секторов для разбиения Рози.

В основе изучения функций $r_{i}(n)$ лежит сведение отображеният $T$ к некоторому повороту окружности, определяемому равенством $x \rightarrow\left\{x+\alpha_{i}\right\}$. Используя компьютерный эксперимент и применяя методы теории чисел, удается установить следующие свойства секторных отклонений $r_{i}(n)$. 
1) Неограниченный рост. $r_{i}(n) \rightarrow \infty$ при $n \rightarrow \infty$.

2) Квазипериодичность. Пусть $r_{i}^{+}(n)$ и $r_{i}^{-}(n)$ соответственно возможные максимальное и минимальное значения отклонений $r_{i}(n)$. Тогда существует бесконечная последовательность квазипериодов $P_{k}^{(i)}$, для которых $\left|r_{i}^{ \pm}\left(n+P_{k}^{(i)}\right)-r_{i}^{ \pm}(n)\right| \leq 3$ для всех $n$. Таким образом, функции $r_{i}(n)$ очень близки к периодическим. Однако свойство неограниченного роста исключает возможность существования чистых периодов функции $r_{i}(n)$. Квазипериоды $P_{k}^{(i)}$ могут быть вычислены по формуле $P_{k}^{(i)}=C^{(i)} Q_{k}\left(\alpha_{i}\right)$. Здесь $Q_{k}\left(\alpha_{i}\right)$ - знаменатель $k$-ой подходящей дроби к углу поворота окружности $\alpha_{i}$, эффективно вычисляемый по разложению $\alpha_{i}$ в цепную дробь.

3) Рост амплитуды. Пусть $A_{k}^{(i)}$ разность между максимальным и минимальным значениями функции $r_{i}(n)$ при $1 \leq n P_{k}^{(i)}$ Тогда $A_{k}^{(i)}$ растет как сумма неполных частных разложения $\alpha_{i}$ в цепную дробь. Точнее, справедливы неравенства $C_{1}^{(i)}\left(k+\sum_{j=1}^{k} q_{j}\left(\alpha_{i}\right)\right) \leq A_{k}^{(i)} \leq C_{2}^{(i)}\left(k+\sum_{j=1}^{k} q_{j}\left(\alpha_{i}\right)\right)$, где $q_{j}\left(\alpha_{i}\right)$ - j-ое неполное частное разложения $\alpha_{i}$ в цепную дробь. Здесь и далее $C_{j}^{(i)}-$ некоторые величины, не зависящие от $n$ и $k$.

4) Форма графика. Если $1 \leq n \leq P_{k}^{(i)}$, то точки графика функции $r_{i}(n)$ располагаются в некотором параллелограмме, одна из диагоналей которого лежит на ось абсцисс и совпадает по длине с квазипериодом $P_{k}^{(i)}$. При этом точки графика сгущаются в некоторой полосе ширины $A_{k-1}{ }^{(i)}$ вдоль границы параллелограмма и равномерно распределены в оставшейся части параллелограмма.

Следует заметить, что квазипериодичность огибающих, ограничивающих $r_{i}(n)$ сверху и снизу проявляется лишь при наличии больших неполных частных $q_{k}\left(\alpha_{i}\right)$. Так, в случае разбиения Рози, угол поворота $\alpha_{i}$ имеет разложение в цепную дробь $\alpha_{i}=[0 ; 1,5,4,2,305,1,8,2,1,4,6,14,3,1,13, \ldots]$. В разложении выделяется большое неполное частное $q_{5}=305$, которому отвечает знаменатель подходящей дроби $Q_{5}=17105$. Тогда, учитывая, что $C^{(i)}=1$, получаем, что заметный квазипериод для отклонений в первом секторе составляет $P_{5}^{(1)}=17105$.

В случае графа вершин разбиения Пенроуза возникающие углы $\alpha_{i}$ должны выражаться через число $\tau$, разложение которого в цепную дробь является периодическим и имеет вид $\tau=[1 ; 1,1,1, \ldots]$. Это исключает возможность появления квазипериодов секторных отклонений $r_{i}(n)$ для графа вершин разбиения Пенроуза. Кроме того, следует учитывать, что в случае графа вершин разбиения Пенроуза поведение секторных координационных чисел $e(x, n)$ существенным образом зависит от четности/нечетности $n$. Поэтому разумно рассматривать функции $r_{i, 0}(n)=r_{i}(2 n)$ и $r_{i, 1}(n)=r_{i}(2 n-1)$. Оказывается, что многие качественные закономерности поведения функций $r_{i, j}(n)$ не зависят от выбора индексов $i j$ и начальной вершины $x$. Главной особенностью графиков функций $r_{i, j}(n)$ является их асимптотическое самоподобие, выражаемое в сохранении формы графика при изменении масштаба по оси 
$n$ в $\tau^{2}$ раз. Соответствующие графики в различных масштабах изображены на рис. 5. В настоящее время описанное поведение отклонений $r_{i, j}(n)$ еще не получило полного объяснения. Однако следует заметить, что эффекты самоподобия с коэффициентом $\tau^{2}$ достаточно типичны для разбиения Пенроуза. В частности, такие эффекты возникают при построении разбиения Пенроуза путем обобщенных преобразований инфляции-дефляции (см. [27] и имеющиеся там ссылки), а также при изучении послойного роста ряда подграфов графа вершин разбиения Пенроуза [29].
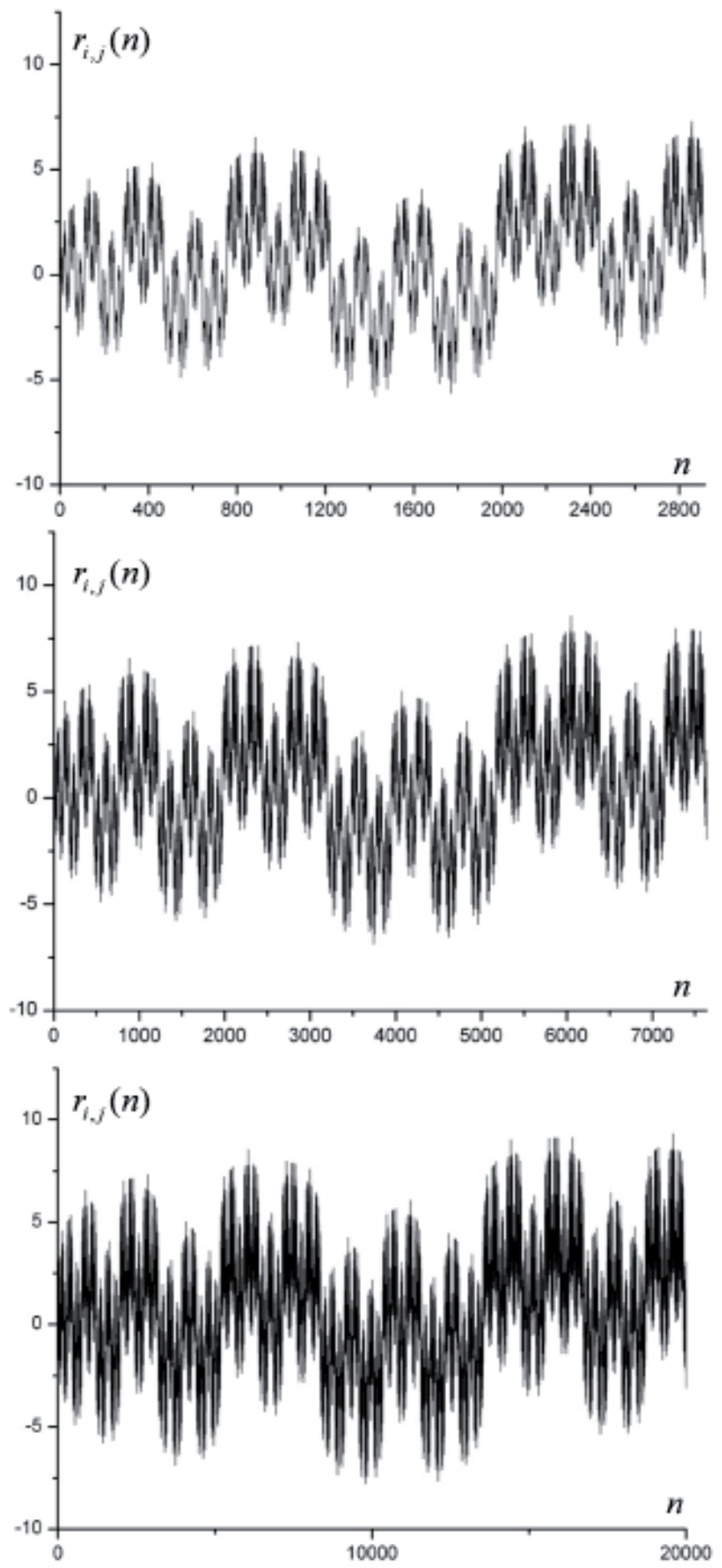

Рис. 5. Асимптотическое самоподобие функиий $r_{i, j}(n)$. 
Неожиданным оказывается также тот факт, что в отличие от разбиения Рози, в случае графа вершин разбиения Пенроуза при некотором выборе начальной вершины $x$ общие отклонения $r^{(j)}(n)=\sum_{i=0}^{9} r_{i, j}(n)$ могут обладать дополнительной структурой, не наблюдаемой на графиках секторных отклонений $r_{i, j}(n)$. В настоящее время такие эффекты обнаружены экспериментально в случае, когда в качестве начальной точки $x$ выбирается начало координат $O$. На рис. 6 изображены графики соответствующих функций $r^{(0)}(n)$ и $r^{(1)}(n)$.
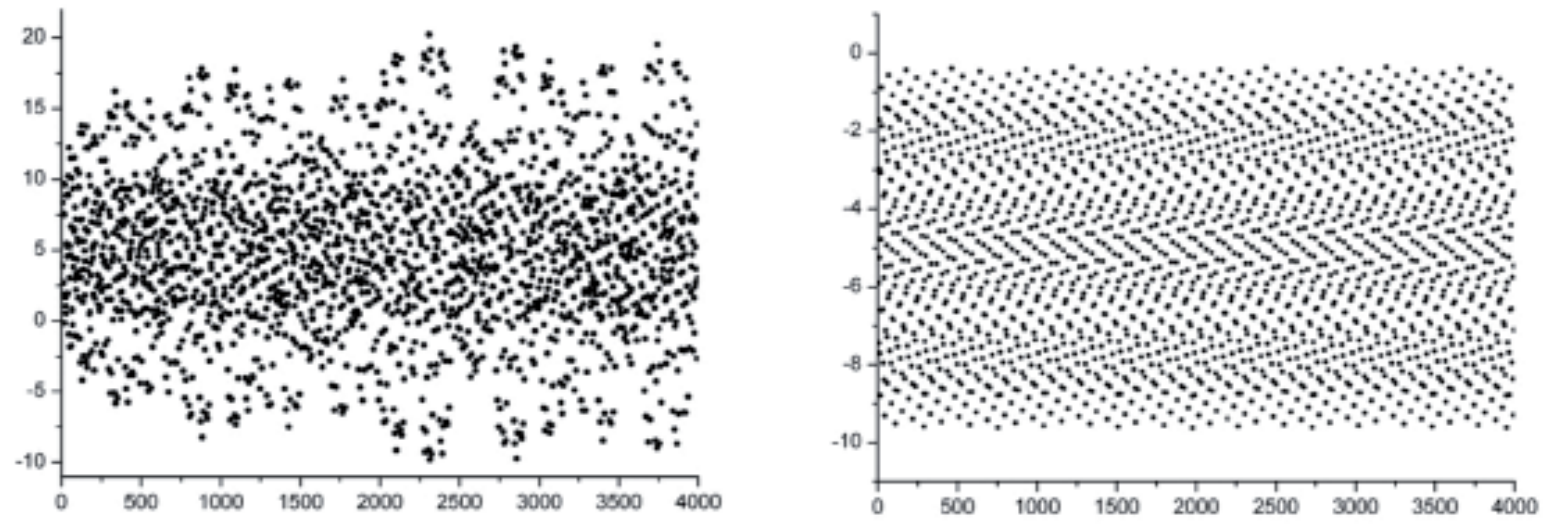

Рис. 6. Графики функциий $r^{(0)}(n)$ и $r^{(1)}(n)$.

Для лучшего понимания структуры графика $r^{(1)}(n)$ разобьем все множество натуральных чисел на 2 множества, задаваемые условиями $\left\{(n-1) \tau^{-2}\right\} \in\left[0 ; \tau^{-1}\right)$ и $\left\{(n-1) \tau^{-2}\right\} \in\left[\tau^{-1} ; 1\right)$ (выбор данных условий объясняется видом случаев 1$)$ и 2$)$ асимптотической формулы для $c(O, n)$. На рис. 7 изображены графики ограничения функции $r^{(1)}(n)$ на эти множества.

Каждый из этих графиков можно рассматривать как часть решетки, заключенную в полосу ограниченной ширины. Соответственно общий график функции $r^{(1)}(n)$ получается наложением двух различных решеток, каждая из которых расположена в своей полосе.
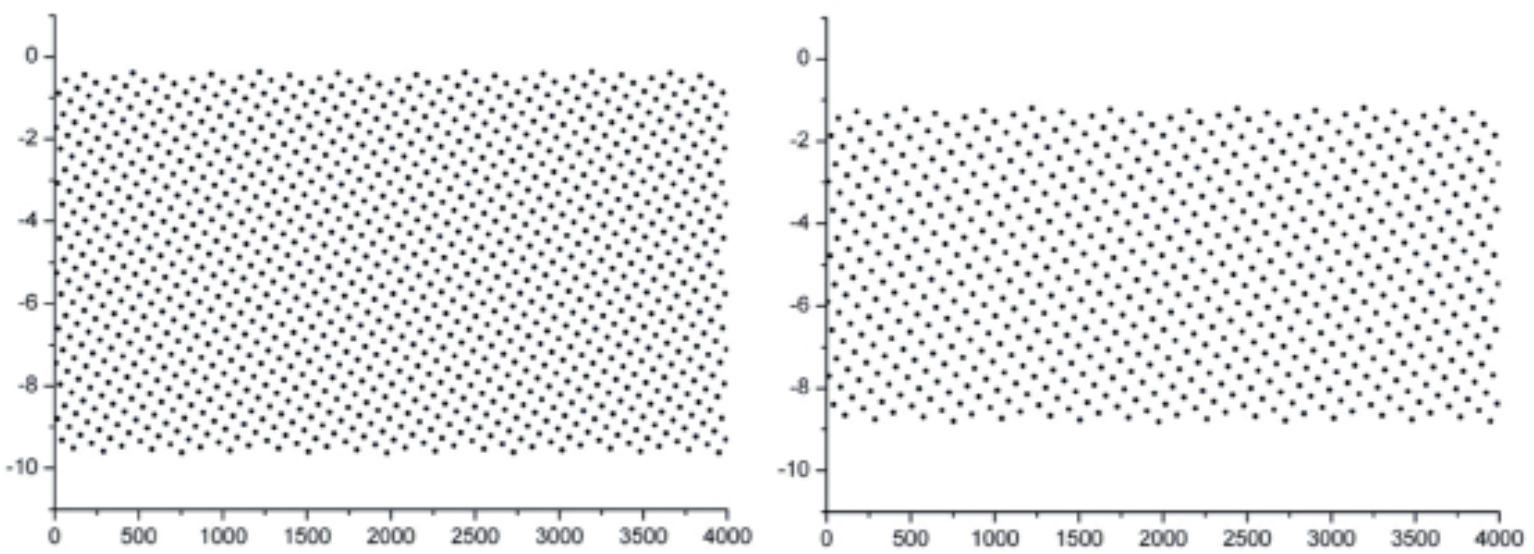

Рис. 7. Графики ограничений функции $r^{(I)}(n)$ на множества, задаваемыле условиями $\left\{(n-1) \tau^{-2}\right\} \in\left[0 ; \tau^{-1}\right) u\left\{(n-1) \tau^{-2}\right\} \in\left[\tau^{-1} ; 1\right)$. 

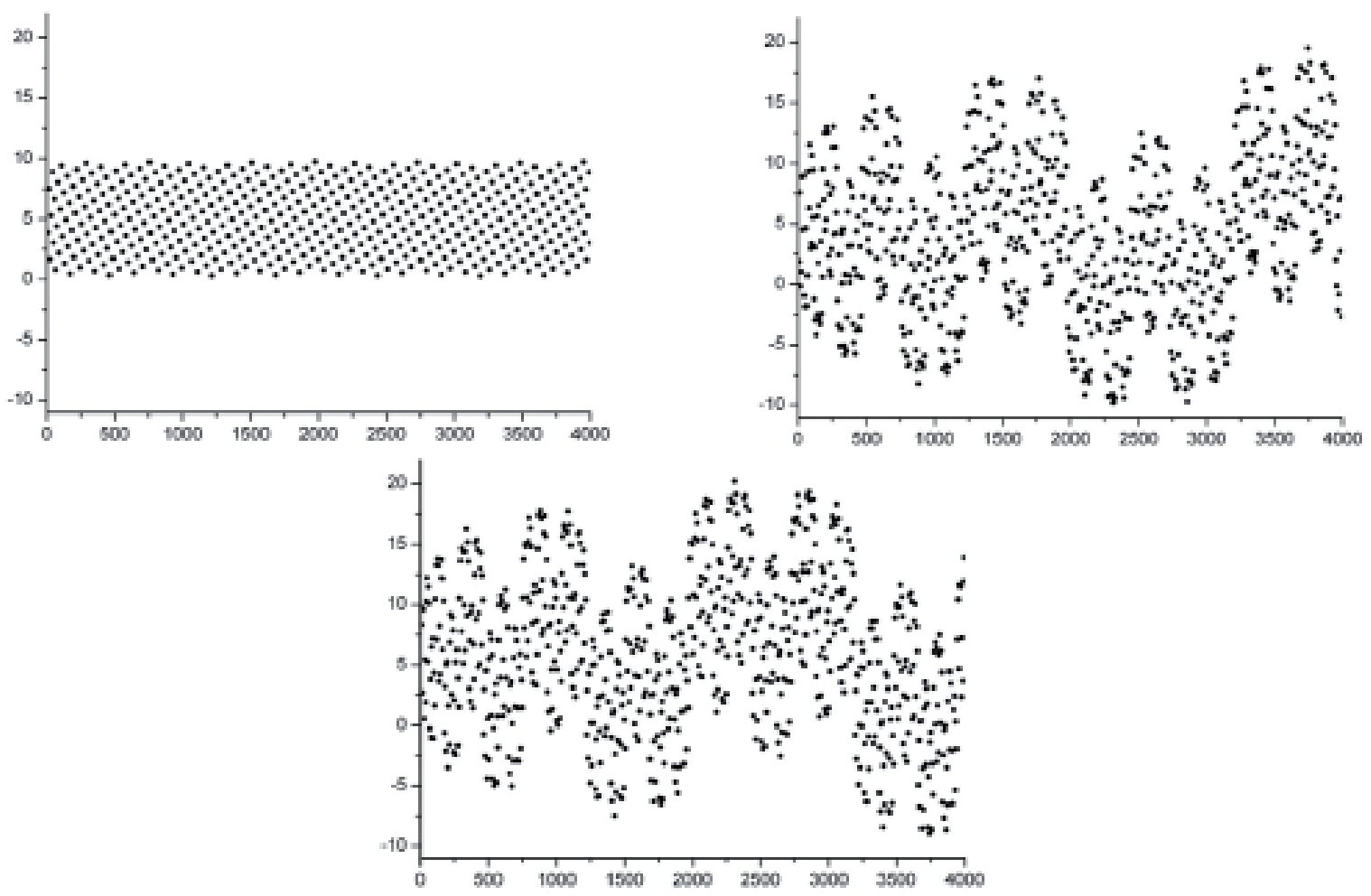

Рис. 8. Графики ограничений функции на множества, задаваемые условиями, $\left\{(n-1) \tau^{-2}\right\} \in\left[0 ; \tau^{-3}\right),\left\{(n-1) \tau^{-2}\right\} \in\left[\tau^{-3} ; \tau^{-1}\right) u\left\{(n-1) \tau^{-2}\right\} \in\left[\tau^{-1} ; 1\right)$.

Аналогично разбивая все множество натуральных чисел на 3 множества, соответствующих случаям 3)-5) асимптотической формулы для $c(O, n)$, получаем, что график функции $r^{(0)}(n)$ представляет собой объединение решетки, расположенной в полосе ограниченной ширины, и двух графиков медленно растущих функций, устроенных аналогично секторным отклонениям $r_{i, j}(n)$ (см. рис. 8).

Интересно, что в обоих случаях появление решетки отвечает ситуации, когда коэффициенты $c(O, n)$, описывающие главные член асимптотической формулы для координационных чисел, представляют собой константы, не зависящие от $n$. Подобных констант не возникает при рассмотрении секторных координационных чисел. При этом существование таких констант в настоящее время получается только как результат весьма громоздких вычислений, не объясняющих при этом возникновение решеток в графиках функций $r^{(j)}(n)$. Поэтому описание функций отклонения остается открытой проблемой, решение которой видимо связано с поиском дополнительных скрытых симметрий между секторами в графе вершин разбиения Пенроуза.

Работа выполнена при финансовой поддержке РФФИ, гранты 17-02-00835-а и 17-42-330787.

\section{Список литературы}

1. Brunner G.O., Laves F. Wiss // Z. Tech. Univ. Dresden. 1971. V. 20. P. 387-390.

2. Meier W.M., Mock H.J. // J. Solid State Chem. 1979. V. 27. P. 349-355.

3. Fischer W. // Z. Kristallogr. 1973. V. 138. P. 129-146. 
4. Fischer W. (1974). Z. Kristallogr. V. 140. P. 50-74.

5. Atlas of Zeolite Structure Types 4th ed., edited by W.M. Meier, D.H. Olson \& C. Baerlocher. Amsterdam: Elsevier. 1996.

6. Шутов А.В., Малеев А.В., Журавлев В.Г. Модель послойного роста разбиений и графов // Tp. V Всерос. науч. школы «Математические исследования в естественных науках». Апатиты, 12-14 октября 2009 г., Апатиты: K \& M, 2009, C. $126-130$.

7. Brunner G.O. // J. Solid State Chem. 1979. V. 29. P. 41-45.

8. Herrero C.P. // J. Chem. Soc. Faraday Trans. 1994. V. 90. P. 2597-2599.

9. O'Keeffe M. // Z. Kristallogr. 1991. V. 196. P. 21-37.

10. O'Keeffe M. // Acta Cryst. 1991. A 47. P. 748-753.

11. Conway J.H., Sloane N.J. A. Low-dimensional lattices. VII. Coordination sequences // Proceedings of the Royal Society A. Mathematical, Physical and Engeneering Sciences. 1997. V. 453, 1966. P. 2369-2389.

12. Baake M., Grimm U. Coordination sequences for root lattices and related graphs // Z. Kristallographie. 1997. V. 212. P. 253-256.

13. Baake M., Grimm U., Repetowicz P., Joseph D. Coordination Sequences and Critical Points // Proceedings of the 6th International Conference on Quasicrystals. Eds. S. Takeuchi and T. Fujiwara. World Scientific, Singapore. 1998. P. 124-127. 14. Рау В.Г., Пугаев А.А., Рау Т.Ф. Координационные числовые последовательности и координационные волны в среде // Кристаллография. 2006. T. 51. № 1. С. 8-16.

15. Grosse-Kunstleve R.W., Brunner G.O., Sloane N. J. A. Algebraic Description of Coordination Sequences and Exact Topological Densities for Zeolites // Acta Cryst. 1996. A. 52. P. 879-889.

16. Eon J.-G. Algebraic determination of generating functions for coordination sequences in crystal structures // Acta Cryst. 2002. A. 58. P. 47-53.

17. Eon J-G Topological density of nets: a direct calculation // Acta Cryst. 2004. A. 60 . P. 7-18.

18. Шутов А.В.Число слов заданной длины в плоских кристаллографических группах // Записки научных семинаров ПОМИ. 2004. Т. 302. С. 188-197. 19. Малеев А.В., Шутов А.В. Модель послойного роста разбиений, упаковок и графов. Владимир, Транзит-Х. 2011. 107 с.

20. Журавлев В.Г. Самоподобный рост периодических разбиений // Алгебра и анализ. 2001. Т. 13. Вып. 2. С. 1-26.

21. Bell E.T. Interpolated denumerants and Lambert series // Am.J. Math. 1943. V. 65. P. 382-386.

22. Beck M., Robins S. Computing the Continuous Discretely, Integer-point enumeration in polyhedra. New York: Springer-Verlag, 2007.

23. Журавлев В.Г., Малеев А.В. Послойный рост квазипериодического разбиения Рози // Кристаллография. 2007. Т. 52. № 2. Р. 204-210.

24. Shutov A.V., Maleev A.V. Quasiperiodic plane tilings based on stepped surfaces // Acta Cryst. 2008. A. 64. P. 376-382. 
25. Шутов А.В., Малеев А.В. Разбиение Пенроуза - модель квазикристаллов // Математические исследования в естественных науках // Тр. XI Всерос. науч. школы. Апатиты, Геологический институт КНЦ РАН, Кольское отделение РМО, 11-12 ноября 2014 г. Апатиты: Изд-во К \& М. 2014. С. 152-161. 26. Шутов А.В., Малеев А.В. Исследование разбиения Пенроуза методом параметризации // Кристаллография. 2018 (принято к публикации).

27. Shutov A.V., Maleev A.V. Penrose Tilings As Model Sets // Crystallography Reports. 2015. V. 60. № 6. P. 797-804.

28. Шутов А.В., Малеев А.В. Сильная параметризация и координационные окружения графа вершин разбиения Пенроуза // Кристаллография, 2017. T. 62. № 4. С. 535-542.

29. Шутов А.В., Малеев А.В. Послойный рост графа вершин разбиения Пенроуза // Кристаллография. 2017. Т. 62. № 5. С. 707-715.

30. Журавлев В.Г., Малеев А.В. Квазипериоды послойного роста разбиения Рози // Кристаллография. 2008. Т. 53. № 1. С. 5-12. 